\title{
Sensory-motor integration training for students with attention-deficit/hyperactivity disorder in Taiwan
}

\author{
Shin-siung Jung* \\ Taipei Institute of Learning Disability, Everspring Foundation, Taiwan
}

\begin{abstract}
Objective: In Taiwan, many parents of children with attention-Deficit/hyperactivity disorder (ADHD) are hesitant about choosing pharmaceutical treatment. As a non-pharmaceutical alternative, we explored the effects of 3 months of Sensory-Motor Integration Training (SMI-Tx) in elementary school students with ADHD.

Method:We compared scores on the ADHD Test (ADHDT), the Sensory-Motor Integration (SMI) checklist, the Test of Nonverbal Intelligence (version 2: TONI2), and the Teacher Rating Scale (TRS) in 94 students with ADHD before and after 3 months of SMI-Tx.

Results: With the exception of students suffering from cold/influenza and neuritis pain, we found that SMI-Tx improved ADHD symptoms in all 94 students. Teacher ratings indicated that ADHD symptoms improved by 79\%. Higher frequency of SMI-Tx sessions was associated with greater improvement in ADHD symptoms.
\end{abstract}

Conclusion: SMI-Tx represents an effective non-pharmaceutical treatment for students with ADHD. However, further studies with larger cohorts and a doubleblind crossover design are needed.

\section{Introduction}

For an accurate diagnosis of attention-deficit/ hyperactivity disorder (ADHD), a child must exhibit six or more symptoms of inattention and six or more symptoms of hyperactivity, impulsivity, and/or distractibility for a minimum of 6 months. Most children present with a form of ADHD that combines these elements, which appear noticeably more often than in other children of the same age.

Children with ADHD frequently encounter problems with temperament, motor skills, learning skills, and social skills, both at home and in the classroom. While the causes of ADHD are still unclear, research suggests that genes, physiological or biochemical malfunctions, brain injury, and environmental factors during pregnancy (i.e., smoking or drinking alcohol) can all play a role. An earlier study with a 33year follow-up assessment period reported that men diagnosed with ADHD as children had significantly worse educational, occupational, economic, and social outcomes [1].

People diagnosed with ADHD are commonly treated with pharmaceutical regimens of methylphenidate (Ritalin: psychostimulant), amphetamine, dextroamphetamine (Adderall: psychostimulant), or atomoxetine (Strattera: a selective norepinephrine reuptake inhibitor) in addition to receiving psychological support and behavioral modification therapy [2]. In our experience, psychological and behavioral treatments often produce acceptable short-term results but may not have long-term effects.

To the best of our knowledge, only one study has reported on the prevalence, incidence, and medication of individuals with ADHD in Taiwan. Conducted from 1996 to 2005, this national insurance population-based study reported that the annual incidence of ADHD diagnosis had increased from $0.02 \%$ to $0.34 \%$ and that stimulant use in children with ADHD had increased from $39.6 \%$ to $54.0 \%$. Both rates were lower than those reported in other community studies [3]. In 2013, Pan, a prominent psychiatrist, stated in a press conference for the Taiwanese Society of Child and Adolescent Psychiatry that only 1 in 20 children with ADHD received proper stimulant treatment [4].

Some parents in Taiwan are quite hesitant to initiate pharmaceutical treatments in their children with ADHD. Treatments involving nonpharmaceutical interventions, such as specialized exercise, may therefore be a viable alternative. Indeed, exercise has been found to improve behavioral, neurocognitive, and scholastic performance in children with ADHD [5]. There is growing interest in alternative therapies for ADHD that involve exercise, such as sensory-motor integration training (SMI-Tx). Such activities are promising because they appear to improve prefrontal function [6] and increase activation in the frontal neocortical association pathway (Figure 1). In this study, we evaluated the effect of SMI-Tx on elementary school aged children with ADHD.

\section{Methods}

\section{Participants}

Over the past 14 years, the treatment unit at the Everspring Foundation has maintained records of 710 students, (533,75\%) boys, $(177,25 \%)$ girls who sought treatment for ADHD or other syndromes. However, there were numerous cases in which the pre- or post-training evaluations by the parents and class teachers were incomplete. In the

${ }^{\star}$ Correspondence to: Shin-siung Jung, Taipei Institute of Learning Disability, Everspring Foundation, Taipei, Taiwan, Email: ssjung@ms2.hinet.net

Key words: ADHD, SMI-Tx, psychostimulant, dyspraxia, nonverbal intelligence

Received: June 05, 2018; Accepted: June 19, 2018; Published: June 23, 2018 

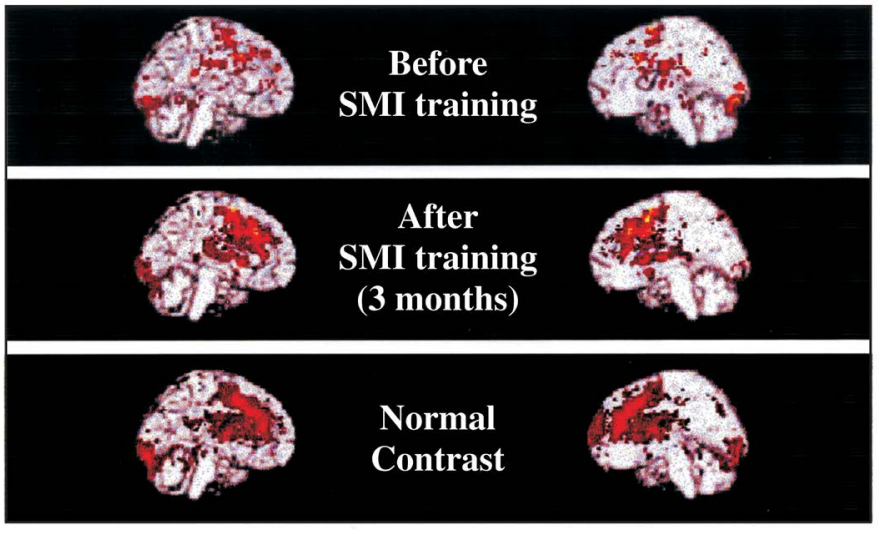

Adapted with permission from Taiwan Special Education Quarterly 101, 2006.

Figure 1. fMRI BOLD Test of Students with ADHD.

1: The upper two images show low activation in the frontal and prefrontal cortical vessels, nerves, and biochemical material in students with ADHD before SMI-Tx. These students were distracted, impulsive, clumsy, emotional unstable, disorganized and exhibited poor execution. The picture is combination of 8 students in the same conditions.

2: The middle two images show moderate levels of activation in the frontal and prefrontal cortical vessels, nerves, and biochemical material. The pathways approach normal levels in students with ADHD after 3 months of SMI-Tx. These students exhibited full concentration and were coordinated, emotional stable, partially organized, and had fair execution. The picture is combination of 8 students in the same conditions.

3: The lower two images show normal levels of activation in the frontal and prefrontal cortical pathways in normal students without SMI-Tx. These students exhibited full concentration and were coordinated, emotional stable, fully organized, and had good execution. The picture is combination of 8 students in the same conditions.

4: In conclusion: During the first stage, we consider what kind of spaces are the frontal and prefrontal pale color bags stage in BOLD fMRI. It should be scanty bags with a few old vessels attracting new vessels first and later nerve sparing technique to establish new territories.

Later reviewing strong exercises (such as SMI-Tx) of muscles and nerves can immediately spreading information to whole body and to brain,and lots of adult have a feeling hot in frontal forehead, and temper immediate come down, indicating sprout nerves go first. Therefor all ADHD symptom disappear in 2 week $\sim 3$ month.

4 years leading up to December 2013, 115 cases (16\%) with complete datasets were available for analysis. Ninety-four of these cases were grade-school students (82\%) with $\mathrm{ADHD}$, and 21 (18\%) were kindergarten pupils with ADHD. As the kindergarten pupils were evaluated using checklists that were different than those used for the grade-school students, we focused our analysis on the 94 grade-school students with ADHD.

Thus, we evaluated the effects of SMI-Tx exercises on 94 students with $\mathrm{ADHD}$ ( 71 males $76 \% ; 23$ females $24 \%$ ) who ranged in age from 7.5 to 10.1 years when they began treatment. The data were collected from December 2009 to December 2013. Before commencing the therapy, parents and class teachers evaluated the children, and we performed a uniform follow-up assessment after 3 months of SMI-Tx.

After admission to our study, 5 of the 94 students dropped out (5\%) after SMI-Tx for 2 months, mainly due to interpersonal conflicts between the spoiled students and their nagging parents, regarding the number of exercises and correction of posture. There were 5 students with ADHD (5\%) taking Ritalin on admission to SMI-Tx program. We did not make any comments or prohibition of taking Ritalin on period of training; 4 (80\%) of them quitted Ritalin on mid-way of training. One male $20 \%$ student kept on Ritalin during 2 months of SMI-Tx and still keep on it 2 years later on telephone interview. Father is of rigid personality trait, and mother is of nagging and histrionic personality. Above 10 students included in this study.

The Therapeutic Ethics Committee at the Everspring Foundation approved this study (SMI-Tx 14-Years Review No. 4). Verbal informed consent was obtained from parents of participants, including those who were videotaped, along with written consent from their parents.

\section{Screening instruments}

We recruited students who had behavioral problems at home and in school. For admission to the training program, students were evaluated by their school teachers and parents using the following four established evaluation instruments: the ADHD Test (ADHDT) [7], the Sensory-Motor Integration (SMI) checklist [8], the Test of Nonverbal Intelligence (version 2: TONI-2) [9], and the Teacher Rating Scale (TRS) (Table 1). Levels of maladjusted behavior at schools were assessed via the 21 sub-items of the TRS. At home, the students were assessed using the 36 sub-items of the ADHDT-C (Chinese version of ADHDT) and 64 sub-items of the SMI checklist (see below).

\section{ADHDT-C}

Parents were asked to complete a 36-item ADHD checklist that we adapted from the ADHDT and translated into Chinese (ADHDT-C). We used the definition of ADHD from the American Psychiatric Association DSM-IV [10], which states that ADHD is characterized by three main symptoms: hyperactivity, impulsivity, and attention deficit. The ADHDT-C has been successfully used at the Everspring Foundation in Taipei for over 4 years. We find that it has excellent sensitivity to ADHD symptoms and is highly consistent with clinical observations. The resulting data had a Cronbach's alpha of 0.941 , and the coefficient of test-retest reliability for a 2-week interval ranged from 0.85 to 0.94 . We used factor analysis to calculate the construct validity of the ADHDT-C, which showed that the KMO (KaiserMeyer-Olkin) measure of sampling adequacy was 0.893 and the result of the Bartlett Test of Sphericity was $2614.16(P=0.0001)$, indicating that the ADHDT-C is adequate for factor analysis. We extracted three elements of construct validity, namely, distractibility (alpha $=0.878$ ), hyperactivity $($ alpha $=0.872)$, and impulsivity $($ alpha $=0.695)$. We used the ADHD-C for admission and for the 3 months post-SMI-Tx evaluation. All students with ADHD had experienced symptoms for a minimum of 6 months (as per the criteria of the National Institute of Mental Health [11]). We compared ADHDT-C scores obtained before and after SMI-Tx therapy.

\section{SMI checklist}

Parents were asked to complete the SMI checklist, which was based on the Ayres checklist [12]. We added items to address observations specific to people in Taiwan regarding deficits in neurophysiological control. The checklist has 64 sub-items in eight subcategories, which are rated on a five-point scale. The eight subcategories are as follows: (1) vestibular dysfunction; (2) deficits in frontal control; (3) sensory processing disorder; (4) childhood dyspraxia; (5) visual form and space disorders; (6) gravitational insecurity; (7) recent temper tantrums or low grades resulting from cold/influenza-induced neuritic pain; and (8) degrees of self-esteem or self-confidence. The resulting data had a Cronbach's alpha of 0.931 and a coefficient of test-retest reliability for a 2-week interval of 0.827 . The sensitivity of the SMI checklist was $98 \%$ (95\% confidence interval [CI] 94-99\%), and its specificity was $94 \%$ (95\% confidence interval [CI] 90-96\%) [8].

We used the SMI-checklist to evaluate the degrees of frontal cortical hypersensitivity (subcategories 2 and 3) and clumsiness (subcategories 1,4 , and 5), which are thought to be associated with a control deficit, and thus may be features of ADHD that could be modulated by SMITx. We compared SMI-checklist results obtained before and after SMITx therapy for 3 months. 
Table 1: Teacher Rating Scale (rate of symptom improvement)

\begin{tabular}{|c|c|c|c|}
\hline & Behavioral Presentations in School Life & $* / * *$ & Improved \% \\
\hline 1 & Talks a lot, endless speech $\quad$ XXX & $29 / 66$ & $44 \%$ \\
\hline 2 & Impulsive; irritable & $46 / 53$ & $87 \%$ \\
\hline 3 & Interrupts others' speech & $34 / 58$ & $59 \%$ \\
\hline 4 & Demonstrates attack behavior & $28 / 28$ & $100 \%$ \\
\hline 5 & Gets frustrated easily & $36 / 63$ & $57 \%$ \\
\hline 6 & Creates chaos during class & $30 / 35$ & $86 \%$ \\
\hline 7 & Talks to few people, has few friends, poor peer relations & $25 / 39$ & $64 \%$ \\
\hline 8 & Very sensitive to environment; cannot tolerate changes in plans or results & $39 / 56$ & $70 \%$ \\
\hline 9 & Clumsy movements, keeps falling down & $29 / 29$ & $100 \%$ \\
\hline 10 & Movements slow and slack, not active, not efficient & $30 / 61$ & $49 \%$ \\
\hline 11 & Reacts strongly to the touch of others & $40 / 49$ & $82 \%$ \\
\hline 12 & Untidy desk and schoolbag & $28 / 57$ & $49 \%$ \\
\hline 13 & Difficulty following class teacher's instructions & $39 / 56$ & $70 \%$ \\
\hline 14 & Frequently forgets to bring books or reports to school & $45 / 56$ & $82 \%$ \\
\hline 15 & $\begin{array}{l}\text { Easily distracted, poor concentration, restless } \mathrm{XXX} \\
\text { while attending class, glancing left and right }\end{array}$ & $74 / 87$ & $85 \%$ \\
\hline 16 & Difficulty with dictation or listening and writing, does not easily understand conversational speech & $26 / 30$ & $87 \%$ \\
\hline 17 & Very clumsy at manual labor in the school and at home & $32 / 34$ & $94 \%$ \\
\hline 18 & Jumps words, lines, or paragraphs while reading & $29 / 42$ & $69 \%$ \\
\hline 19 & Great difficulty in understanding questions and transcribing formulas in mathematics & $28 / 48$ & $58 \%$ \\
\hline 20 & Frequently jumps words, lines, or paragraphs when copying & $31 / 44$ & $70 \%$ \\
\hline 21 & Eyes drift while in class, not able to concentrate fully & $30 / 68$ & $44 \%$ \\
\hline \multirow{2}{*}{\multicolumn{2}{|c|}{$\begin{array}{l}\text { *Number of students who improved on this sub-item after } 3 \text { months of SMI-Tx } \\
* * \text { Number of students with defects on this sub-item }\end{array}$}} & \multicolumn{2}{|c|}{ Mean $=72 \%$} \\
\hline & & \multicolumn{2}{|c|}{$\mathrm{SD}=18 \%$} \\
\hline
\end{tabular}

Note: 1 . We assessed a total of 94 students with ADHD over a 4 year period. 2. XXX indicate 4 symptoms of ADHD with mean of improvements $79 \%$.

\section{TONI-2}

We asked the teachers at the Everspring Foundation to complete the TONI-2, which is a nonverbal intelligence test used to assess the IQ of non-English-speaking children and adults aged 5-85 years. Using pictures, patterns, and geometry to test deductive and analytical reasoning skills, the examiner does not speak, but rather gestures and points to ask questions. The coefficients of test-retest reliability for a 2 -week interval ranged from 0.80 to 0.95 . Normal performance on the TONI-2 is a score of 100 . Individuals with scores in the 60 s are considered to have an intellectual developmental disorder, while gifted subjects achieve mean scores of approximately 115 . Individuals with LDs, dyslexia, and mild intellectual deficits, as well as students referred to psychological services as a result of poor academic performance, tend to score in the 80s and 90s [9].

We used the TONI-2 to assess general nonverbal mental ability. This enabled us to screen out students with below-average mental abilities. We compared TONI-2 scores obtained before and after SMITx therapy.

TRS for the assessment of maladjustment at school

The TRS (Table 1) was designed by the chief of education services at the Everspring Foundation. A teacher uses a five-point scale (Never-1, Few-2, Occasionally-3, Frequently-4, Always-5) to rate the frequency of 21 daily emotions and behaviors of their students. We asked the teachers to complete a pre-test TRS after observing the students for 3-4 weeks at the beginning of a new semester, and a post-test TRS 1-2 weeks before the end of a semester. Each class included approximately 20-30 classmates. High ratings correspond to more severe adjustment difficulties. The coefficient of test-retest reliability for a 2-week interval was 0.865 . We used factor analysis to calculate the construct validity of the TRS, which showed that the KMO measure of sampling adequacy was 0.835 and the result of the Bartlett Test of Sphericity was 1079.803 $(P=0.0001)$, indicating that the TRS is adequate for factor analysis. We extracted three elements of construct validity: sensory processing disorder $(\mathrm{SPD})($ alpha $=0.860)$; eye-drifting dysmetria $($ alpha $=0.852)$; and dyspraxia, clumsiness, or poor coordination (alpha $=0.732$ ).

We used the TRS, completed by class teachers, to evaluate factors associated with ADHD (items 1, 2, 4, and 15). Additionally, we assessed items $18,19,20$, and 21 , which concern eye drift, and thus may be related to inattention as well as difficulties with visual learning. These are judged best by class teachers. We compared TRS scores obtained before and after SMI-Tx therapy for 3 months.

\section{Intervention methods}

SMI-Tx is a series of exercises mainly involving prone extension postures. These postures are thought to be beneficial for treating individuals with general hypersensitivity, as well as those with control deficits in attention, impaired motor coordination, and sensory processing disorders. Specifically, the exercises that comprise SMITx are thought to enhance kinesthetic and vestibular impulses, thus activating the neocortical association pathway in the prefrontal lobes [6]. As this region corresponds to cortical dysfunction in people with $\mathrm{ADHD}$, enhanced activation has the potential to facilitate positive behavioral change.

The SMI-Tx exercises were introduced to the parents and students via videos and actual practice sessions. The exercises were performed to a count of 50 beats, which were spoken out loud by the player, and included pushing a ball against a wall, exchanging a ball with an adult who was in a sitting position, and moving by "walking” on one's hands. All of these actions were performed while lying prone on a plywood scooter board $(47 \times 30 \mathrm{~cm})$, which was covered in coarse carpet and mounted on 4-directional iron wheels. 
During the first week of treatment, students in grades 1,2 , and 3 were assigned daily walking-on-hands, ball-pushing, and ballexchanging exercises (using a dodge-ball) for a count of 100 beats. The count was increased by 50 for a total of 150 beats in the second week, increased by another 50 counts for a total of 200 beats in the third week, and so on until a maximum daily count of 400-600 beats was reached. The number of beats was increased weekly to gradually build up body strength.

The daily exercises could be divided into two or three sessions as needed, based on fatigue, complaints of pain, disengagement, or similar problems. Towards the end of the breaks between sessions, parents had the option of offering the students water, fruit juice, vitamin B complex, or a small piece of bread. Students in the $4^{\text {th }}$ grade and above used basketballs for the exercises and started with an initial daily count of 200 beats. As with the younger students, the number of beats increased by 100 each week, with a maximum daily count of approximately 600-800 beats for each exercise. These numbers were meant as general guidelines, and supervising teachers or parent volunteers could modify them according to the body weight or sex of the children.

When students practiced SMI-Tx in groups, they often competed with one another. As a result, the number of beats for each exercise could easily reach $600-800$ per session, which was likely associated with increased efficacy. A video showing the individual and school group exercises may be accessed at:

h t t p s : / / d rive.google.com/f i l e / d/0B5J5qR2tY95iRGVNUUVHTkRGMWs/view? usp=sharing

Participants completed an average of $2.2 \pm 0.1$ SMI-Tx sessions at the Foundation per week. However, 37 of the students (40\%) completed 3-4 sessions at the Foundation per week. After encouragement, 59 students $(62 \%)$ agreed to complete three types of weekly exercises at home for a total of 5-7 exercise sessions per week. The average length of training at the Foundation was $4.0 \pm 2$ months.

Based on previous experience, we considered fewer than two SMITx sessions per week to be insufficient for noticeable improvement. Therefore, we divided our sample into two groups: participants who had completed the SMI-Tx exercises just once per week (eight students, exercises completed mainly at the Foundation) and participants who had completed the SMI-Tx exercises multiple times per week, both at the Foundation and at home (86 students). We analyzed group membership as an independent variable and scores on the SMI checklist, ADHDT-C, and TRS as dependent variables. The general analysis included all 94 students.

\section{Results}

\section{Statistical analyses}

A total of 94 elementary school students with ADHD completed 3 months of SMI-Tx, with complete datasets for the ADHDT, SMI checklist, TONI-2, and TRS before and after treatment.

A paired $t$-test revealed that the post-training scores on the TONI-2 (mean $=121.5)$ were significantly higher than the pre-training scores (mean $=114.1 ; P<0.001)$. In addition to the 3 month assessment, we conducted evaluations 6 months post SMI-Tx for 3 students with borderline TONI-2 scores (i.e., scores ranging from $80-88$ ), which had improved to $90-92$ by 6 months, and 9 months post SMI-Tx for one student of slow developer with a TONI-2 score that eventually rose from 86 to 115 .
Furthermore, Student's $t$-tests showed that scores for the nine SMI checklist subcategories (eight syndromes + sum), ADHDT-C symptoms (both based on parental evaluations), and the three TRS syndromes (based on teacher evaluations), all showed significant improvement after 3 months of SMI-Tx $(P<0.001$, Table 2$)$. These data indicate that the parent and teacher evaluations captured improved ADHD behaviors after 3 months of SMI-Tx, which may have reflected changes in brain function suggested by the pre- and post treatment results of the TONI-2.

We used a two-factor mixed design multivariate analysis of variance (MANOVA) to assess the changes in TRS and ADHDT-C syndromes. We found a significant effect of frequency of training on test scores (TRS: F1,86 = 9.09; ADHDT-C: F1,86 = 13.43, $P<0.05$ ), indicating that students who engaged in multiple sessions per week showed significantly greater improvement. However, further analysis revealed no interaction between frequency group ( 1 time per week vs. 2 or more times per week) and test score (TRS: F1,86 = 1.08; ADHDT-C: F1,86= $0.33, P>0.05)$, indicating that the differences in scores between the two groups were approximately the same for each test. We then conducted a MANOVA with a two-factor mixed design that included the SMI syndromes (eight syndromes from the SMI checklist), which revealed an interaction between the groups and the tests $(\mathrm{F} 1,86=3.08 ; P<0.05)$, indicating that this issue requires further clarification.

An analysis of variance (ANOVA) using SMI syndrome as an independent variable revealed that the improvements associated with 3 months of SMI-Tx multiple times weekly were associated with progress in the following subcategories: (1) vestibular dysfunction, (2) frontal control deficit, (3) sensory processing disorder, (4) childhood dyspraxia, (5) visual form and space disorders, (6) gravitational insecurity, and (8) lack of self-esteem and self-confidence $(P<0.05)$. Students who were reported as having (7) recent temper tantrums or low grades resulting from colds or latent influenza did not exhibit a significant improvement as a result of SMI-Tx therapy $(F 1,86=3.914 ; P>0.051)$. However, it seems likely that children with a cold/influenza will exhibit impaired functioning for 1-2 weeks following a cold/influenza, or perhaps an even longer period. Thus, recent regressed behaviors may not be due to resistance to treatment or a lack of active/passive cooperation from students. We encourage parents to be patient with their children and to watch carefully for signs of cold/influenza. In periods of illness (in the absence of a high fever), we suggest limiting SMI-Tx therapy to the high speed ball exchange exercise for 20-30 minutes daily, in addition to offering water, fluit juices, Vitamin B complex, and encouragement. This short exercise may help students maintain a clear mind with high efficiency. We recommend the same procedure when students have limited time for rehearsal owing to mid-terms or final examinations.

Table 1 shows scores on the 21 sub-items of the TRS for the 94 students. Improvement, as rated by class teachers, ranged from $100 \%$ to $44 \%$. We regard the first two items on the scale as indicative of 'normal' behavior (Never and Few). We regard changes in the last three items (Occasionally, Frequently and Always) to normal levels as 'improved'. The improvement rates for the 94 students in terms of items on the TRS are shown in the last two columns of table 1 . There were 9 items with improvement rates over $80 \%$ : 'attack behavior' improved by $100 \%$ (item 4); 'clumsy movements' improved by $100 \%$ (Item 9); 'very clumsy at manual labor in school' improved by $94 \%$ (Item 17); 'difficulty with dictation or listening and writing' improved by $87 \%$ (Item 16); 'impulsive and irritable' improved by $87 \%$ (Item 2); 'creates chaos during class' improved by $86 \%$ (Item 6); 'easily distracted and poor concentration' improved by $85 \%$ (Item 15); 'reacts strongly to the touch 
Table 2: Student $t$-Test results showing improvement on the TONI-2, and SMI(9 syndromes), ADHDT (4 syndromes), and TRS (3 syndromes)

\begin{tabular}{|c|c|c|c|c|c|c|c|c|c|c|c|c|c|c|c|c|c|}
\hline $\mathbf{N}$ & $\mathbf{T}$ & S1 & S2 & S3 & S4 & S5 & S6 & S7 & S8 & S9 & A1 & A2 & A3 & A4 & T1 & $\mathrm{T} 2$ & T3 \\
\hline 1 & 15 & -2 & 6 & -1 & 1 & 1 & -2 & 1 & 6 & 9 & 7 & 7 & 1 & 14 & -3 & -3 & 5 \\
\hline 2 & 7 & 1 & -1 & 3 & -11 & 1 & 5 & 1 & 3 & 1 & 3 & 0 & -2 & 1 & 4 & 0 & -2 \\
\hline 3 & 11 & -2 & 2 & 5 & 1 & -3 & 5 & -1 & 4 & 12 & 5 & 6 & 7 & 19 & 1 & -3 & -1 \\
\hline 4 & 10 & 2 & 3 & 3 & -3 & 1 & 1 & -2 & -1 & 6 & 2 & 2 & -7 & -4 & -1 & 5 & 1 \\
\hline 5 & 11 & 1 & 4 & 10 & 9 & -6 & 7 & 3 & -1 & 24 & -1 & -1 & -8 & -13 & 0 & -2 & 4 \\
\hline 6 & 17 & 6 & -8 & 0 & 10 & 2 & 2 & 0 & 2 & 14 & 0 & 1 & 3 & 4 & -7 & -4 & -7 \\
\hline 7 & 8 & 4 & 8 & 8 & 5 & -1 & 3 & 4 & 4 & 31 & 3 & 7 & 8 & 19 & 2 & -1 & 1 \\
\hline 8 & 17 & 6 & 9 & 5 & 0 & 1 & 2 & -3 & -1 & 22 & -2 & -1 & -6 & -11 & 0 & -1 & 5 \\
\hline 9 & 13 & 4 & 5 & -2 & -10 & -2 & -5 & -1 & 0 & -10 & 4 & 2 & 6 & 13 & -4 & 1 & 3 \\
\hline 10 & -22 & 3 & 4 & -2 & -11 & -3 & 0 & -3 & -2 & -11 & -8 & -7 & 0 & -16 & 7 & 3 & 5 \\
\hline 11 & 5 & -5 & 2 & -1 & -7 & -7 & 0 & -6 & -3 & -21 & -4 & -3 & -6 & -13 & 1 & -3 & -3 \\
\hline 12 & 0 & -9 & -5 & -7 & 1 & -3 & -6 & -2 & 0 & -29 & -3 & -2 & -2 & -8 & -4 & 0 & -5 \\
\hline 13 & -8 & -5 & -8 & -14 & -15 & -9 & -10 & 1 & -3 & -64 & -8 & 1 & -1 & -12 & -2 & -3 & -2 \\
\hline 14 & 0 & 1 & -5 & -17 & -4 & -4 & -4 & -7 & -3 & -36 & -7 & -4 & -1 & -12 & -1 & 3 & -2 \\
\hline 15 & 19 & -5 & -6 & -2 & -1 & -8 & 0 & 0 & 1 & -21 & -6 & -2 & -5 & -14 & -2 & 0 & -3 \\
\hline 16 & 8 & -1 & 2 & -7 & -14 & -7 & -8 & -1 & 0 & -35 & -8 & -9 & -13 & -32 & 0 & 1 & 3 \\
\hline 17 & -5 & -11 & -4 & -10 & -20 & -7 & -18 & -6 & -2 & -72 & -2 & -1 & 3 & 0 & -1 & 0 & 2 \\
\hline 18 & 0 & 2 & -1 & -2 & -2 & -2 & -3 & 0 & 0 & -8 & -1 & 1 & 0 & 0 & -14 & 1 & -2 \\
\hline 19 & 0 & -5 & -11 & -8 & 1 & -1 & -6 & 0 & -1 & -31 & 0 & 5 & 6 & 13 & -3 & 2 & 1 \\
\hline 20 & 10 & 1 & -4 & -13 & -5 & -3 & 0 & 2 & 0 & -24 & -9 & -2 & -2 & -14 & -6 & 3 & 3 \\
\hline 21 & 10 & -10 & -5 & -4 & -3 & 1 & -1 & -2 & -1 & -23 & -5 & -4 & -4 & -14 & -12 & 2 & -6 \\
\hline 22 & 15 & -8 & 2 & -2 & -19 & -4 & -5 & 3 & 2 & -34 & -5 & -1 & -5 & -13 & -5 & 1 & -1 \\
\hline 23 & 19 & -3 & 1 & 1 & -8 & -2 & -15 & -2 & -1 & -27 & 3 & -2 & 2 & 2 & -4 & -2 & -5 \\
\hline 24 & 4 & 3 & -1 & -1 & 1 & -2 & -8 & -1 & 0 & -8 & -4 & 0 & -1 & -11 & -8 & 0 & -5 \\
\hline 25 & 13 & -8 & -1 & -6 & 3 & -2 & -1 & 1 & 2 & -13 & -1 & -2 & -1 & -4 & -2 & -6 & -4 \\
\hline 26 & 20 & -14 & -6 & -15 & -13 & -7 & -10 & -1 & -4 & -69 & -4 & -6 & -3 & -13 & -4 & -4 & -7 \\
\hline 27 & 1 & -6 & -2 & -5 & -4 & -4 & -5 & -1 & -1 & -27 & -7 & -7 & -5 & -20 & 1 & 1 & 5 \\
\hline 28 & 12 & -8 & -6 & -2 & -10 & -1 & -5 & -2 & -1 & -33 & -11 & -7 & -17 & -34 & 0 & 1 & -1 \\
\hline 29 & 2 & -9 & 0 & -1 & -3 & -6 & 1 & 0 & 0 & -18 & -14 & -7 & -10 & -32 & -9 & 2 & -5 \\
\hline 30 & -6 & -20 & -5 & -13 & -24 & -9 & -10 & -4 & 1 & -80 & -11 & -5 & -8 & -26 & -5 & 4 & 7 \\
\hline$t$-Test & $P<$ & 0.001 & 0.001 & 0.001 & 0.001 & 0.001 & 0.001 & 0.001 & 0.001 & 0.001 & 0.001 & 0.001 & 0.001 & 0.001 & 0.001 & 0.001 & 0.001 \\
\hline
\end{tabular}

Note: Total student numbers 94 ; with space limitation, only showed 30 students.

N: Student No., T: Improved scores of TONI-2, S1: Improved vestibular dysfunction, S2: Improved frontal control deficits, S3: Improved sensory process disorder, S4: Improved childhood dyspraxia, S5: Improved form \& space disorder, S6: Improved gravitational insecurity, S7: Improved short temper \& grading, S8: Improved self-esteem \& confidence, S9: Improved sum of SMI syndromes, A1: Improved hyperactivity, A2: Improved impulsion, A3: Improved inattention, A4: Improved sum of ADHDT, T1: Improved score of pan-hypersensitivity \& control deficits, T2: Improved score of eye drift, jumping, poor scholastic scores, T3: Improved score of dyspraxia, clumsiness, \& in-coordination

of others' improved by $82 \%$ (Item 11); and 'frequently forgets to bring books or reports to classes' improved by $82 \%$ (Item 14).

Item 1, which is related to deficient in talking control (e.g., "talks a lot"), and Item 21, which is related to eye concentration and well control (e.g., "eyes drift while in lecture"), together had the lowest rates of improvement at only $44 \%$ at the 3 month follow-up evaluation. This indicates that longer periods of SMI-Tx might be required to obtain greater improvement in those two items. Overall, the mean total improvement rate was $72 \% \pm 18 \%$. The mean improvement for the 4 ADHD symptoms (item 1, item 2, item 4, item 15) on the TRS was $79 \%$.

\section{Discussion}

Table 2 shows the results of the Student's $t$-test, which revealed improvements in TONI-2, ADHDT-C, and TRS scores between the two assessments (spaced 3 months apart). We also found improvements in post-SMI-Tx scores associated with deficiencies in SMI- syndrome $(P$ $<0.001)$.

Our data indicate that intensive SMI-Tx sessions multiple times per week can lead to significant improvements in ADHD-C symptoms. We used the SMI-checklist to evaluate factors associated with the degree of frontal cortical hypersensitivity $(2,3)$ and clumsiness or incoordination $(1,4,5)$, which are associated with control deficits, because we expect that these elements are related to ADHD, and could thus be treated with SMI-Tx. We found significant improvement in these factors, indicating that SMI-Tx had a positive effect.

In terms of the SMI-checklist, we found that the higher the frequency of SMI-Tx sessions per week (5-7 sessions per week), the greater the improvements in ADHD symptoms.

With respect to the TRS (Teacher Rating Scale), we found that the mean improvement in 4 ADHD symptoms (items 1, 2, 4, and 15) after 3 months of SMI-Tx was 79\%. Overall, our findings indicate that frequent SMI-Tx can have a positive effect on ADHD and ADHDrelated symptoms in elementary school students.

\section{BOLD fMRI study of students with ADHD}

In 2006 a research group conducted a BOLD fMRI study of students with ADHD [13] and found that students with ADHD had very limited activation in the frontal regions prior to SMI-Tx. Three months after SMI-Tx, BOLD fMRI revealed increased activation in the frontal and prefrontal regions, and the patterns of activation were more similar to those observed in a group of healthy contrast. According to observations and ratings by teachers and parents, the individuals in this experimental group exhibited improved symptoms in terms of ADHD-C scores as well as improved motor skills and motor execution after 3 months of SMI-Tx (Figure 1). 


\section{The underlying theoretical framework of SMI-Tx}

In our SMI-Tx program, students perform three types of activities in a prone extension position on a scooter board. Exercises involving prone extension postures produce strong kinesthetic nerve impulses from muscles, ligaments, and joint surfaces in face, mouth, nose, ears, eyes, 4 limbs, neck, and back. Maintaining one's balance, even subconsciously, involves focal and large-scale body movements that produce plentiful vestibular and strong kinesthetic nerve impulses that are transmitted immediately to the entire body [14], including the brain.

Cortical brain regions are activated by strong kinesthetic nerve impulses resulting from whole body movements (strong neuron exercises), and new neuronal pathways are established in frontal and prefrontal brain (highest position in position of prone extension posture). The kinesthetic system works to send messages throughout body parts, providing a direct connection to the cortex of the brain. Improved signal transmission in the frontal and prefrontal neuronal pathways decreases hyperactivity, impulsiveness, severe distraction, and eye drifting, improves general hypersensitivity associated with control deficits (sensory processing disorder), and facilitates fine motor control and coordination of hand fingers, mouth, face, eyes, and ears (Figure 1), covering all chief and accessory symptoms and signs of ADHD.

Vestibular system on the other hand, allows us to stand upright (in our prone extension exercise posture, frontal and prefrontal lobes are tip upright posture), maintain balance, and move through space by means of its direct connection to the brain. The vestibular system coordinates information from all of the vestibular organs. These organs include the inner ear, the eyes, the muscles and joints, the fingertips, the palms of the hands, the soles of the feet, pressers of the jaw, and lastly; gravity receptors (which are located on the skin and work to adjust a number of things including heart rate, blood pressure, muscle tone, limb position, immune responses, arousal and balance). Those are call "Brain Training".

If daily morning before going to school and immediate after school before doing homework take 20-40 minutes, doing following mentioned "high-speed ball exchange" play. The classes and home will be much smooth.

\section{Implementing SMI-Tx}

At the Foundation, we emphasize the value of home therapy, which consists of three types of SMI-Tx exercises. To encourage parents to make time for the exercises at home, we provide them with individual and group demonstrations and supporting discussions. We explain that exercises can release endorphins, providing students with feelings of pleasure, happiness, and a sense of accomplishment. We also explain and expect improved activity in neuronal pathways by the third week of training, which will improve initial hyperactivity, irritability, impulsive speech and behavior, and concentration.

Many students with ADHD have very low self-esteem and selfconfidence. Thus, in our discussions with parents, we stress that nagging is very destructive and may produce anxiety and irritability in students with ADHD who suffer from hypersensitivity and control deficits, potentially resulting in passive or active resistance to the exercises. We suggest that parents simply accompany their children while they exercise, accept their weaknesses without correction, and occasionally offer short and substantial words of praise. Improvement in frontal and prefrontal neocortical pathways may alleviate emotional, speech, posture, and behavioral problems.
For very busy students in the higher grades (grades 4-5 and above), we suggest introducing another exercise called the "high-speed ball exchange". We recommend that students engage in this task for 20-30 minutes in the morning before going to school or immediately after school before doing homework. In this task, parents initially gently bounce a ball to the midway point between themselves and their children's hands or chin level, at a distance of approximately 180-200 $\mathrm{cm}$. The child is expected to catch and return the ball to the parent. Within 2-4 weeks, the balls are then aimed toward the child's face and then, finally, to the left and right sides of the child's head in random order for as long as the child can catch the balls. This simple exercise helps students sustain full concentration throughout the day by increasing activation in the frontal and prefrontal neocortex.

For younger students, once they have completed the low-speed ball exchange exercise for 3-5 weeks and are familiar with this task, we suggest that they advance to the high-speed ball exchange. For both younger and older students, the high-speed ball exchange exercise is an effective method for improving symptoms of general hypersensitivity with control deficits (sensory processing disorders) and eye drifting. To view a video of this exercise, please see the following link:

h t t p s : / / d r i ve.google.com/file/ d/0B5J5qR2tY95iZHM5RkkyNDdlOEU/view?usp=sharing

\section{Conclusion}

SMI-Tx represents an effective non-pharmaceutical treatment for students with ADHD. Three months of SMI-Tx led to improvements in ADHD symptoms that were noted by parents and class teachers (Table 1), validated by the TONI-2 (Table 2), and are supported by a previous study that reported corresponding changes in BOLD fMRI activity (Figure 1) before and after 3 months of SMI-Tx. The more intensity of SMI-Tx (5-7/week), the results are quicker and better. However, further studies with larger cohorts and a double-blind crossover design are needed.

\section{References}

1. Klein RG, Mannuzza S, Olazagasti MA, Roizen E, Hutchison JA, et al. (2012) Clinical and functional outcome of childhood attention-deficit/hyperactivity disorder 33 years later. Arch Gen Psychiatry 69: 1295-13303. [Crossref]

2. McCarthy LF (2008) Behavior therapy for ADHD children: more carrot, less stick ADDitude Summer 2008:12-15.

3. Chien IC, Lin CH, Chou YJ, Chou P (2012) Prevalence, incidence, and stimulant use of attention-deficit hyperactivity disorder in Taiwan, 1996-2005: a national populationbased study. Soc Psychiatry Psychiatr Epidemiol 47: 1885-1890. [Crossref]

4. Pan JH (2011) Only 1 in 20 kids with ADHD get proper treatment. Press conference by Taiwanese Society of Child and Adolescent Psychiatry (TSCAP). Taipei: China Post 2011:11,15.

5. Pontifex MB, Saliba BJ, Raine LB, Picchietti DL, Hillman CH (2013) Exercise improves behavioral, neurocognitive, and scholastic performance in children with attention-deficit/hyperactivity disorder. J Pediatr 162: 543-551. [Crossref]

6. Arnsten AF (2009) Toward a new understanding of attention-deficit hyperactivity disorder pathophysiology: an important role for prefrontal cortex dysfunction. CNS Drugs 23: 33-41. [Crossref]

7. Gilliam JE (1995) Attention-deficit/hyperactivity disorder test (ADHDT) Psychological Assessment Resources: 25: 3-165.

8. Jung SS, Lee YC (1991) Manual of child sensory-motor integration (SMI) checklist and norm. In Taipei Special Education Center, Elementary Special Education: Booklet 32: 7-36. (Translated from Chinese).

9. Brown L, Sherbenou RJ, Johnsen SK (1990) The Test of Nonverbal Intelligence, Second Edition. Austin, Texas: PRO-ED Inc. pp. 1-55. 
10. American Psychiatric Association (2000) Diagnostic and statistical manual of mental disorders (fourth ed., text rev.). Washington DC: pp. 83-85.

11. National Institute of Mental Health (2008) Attention deficit hyperactivity disorder (ADHD): A detailed booklet describing ADHD symptoms, causes, and treatments, with information on getting help and coping. U.S. Department of Health and Human Services, National Institutes of Health. National Institutes of Health Publication No. 08-3572.
12. Ayres J (1980) Sensory integration and the child. Los Angeles: Western Psychological Services pp. 81-120.

13. Jess M, Mahtob K, Ashley K (2016) Body movement kinesthetic and vestibular sense. Tangient LLC.

14. Jung SS, Yeh TZ, Loo HH, Tsuan ML, Yan JM, et al. (2006) Efficacy of fMRI in ADHD children with sensory-motor integration training. Taiwan Spec Ed Q 101: 9-16.

Copyright: (2018 Jung S. This is an open-access article distributed under the terms of the Creative Commons Attribution License, which permits unrestricted use, distribution, and reproduction in any medium, provided the original author and source are credited. 